\title{
Correlation of Fibulin-2 expression with proliferation, migration and invasion of breast cancer cells
}

\author{
XILIANG ZHANG $^{1 *}$, LIAN DUAN $^{2 *}$, YUXING ZHANG $^{1}$, HUIBIN ZHAO $^{1}$, \\ XIAODONG YANG ${ }^{1}$ and CHAOJUN ZHANG ${ }^{1}$ \\ ${ }^{1}$ Department of General Surgery, Navy General Hospital, Beijing 100048; ${ }^{2}$ Department of Endocrinology, \\ Peking Union Medical College Hospital, Beijing 100730, P.R. China
}

Received December 3, 2018; Accepted February 4, 2020

DOI: $10.3892 / \mathrm{ol} .2020 .11747$

\begin{abstract}
Expression level of Fibulin-2 gene in breast cancer cells was evaluated to explore the impact of Fibulin-2 gene on the proliferation, migration and invasion of breast cancer cells. MDA-MB-231, BT483, MCF-7 and SK-BR-3 breast cancer cells were cultured in vitro. Then, expression of Fibulin-2 in cells was upregulated and downregulated using ribonucleic acid interference (RNAi) and lentiviral transfection techniques, respectively. Thereafter, expression levels of Fibulin-2 messenger RNA (mRNA) and protein were measured via quantitative real-time reverse transcription-polymerase chain reaction and western blotting, respectively. Cell Counting Kit-8 assay was applied to detect the proliferation ability, and wound healing assay was performed to determine the effect of transfection on the metastatic capacity of cells. The influence of transfection on the invasive ability of breast cancer cells was detected through Transwell chamber assay. MDA-MB-231 and MCF-7 cells did not express Fibulin-2, while BT483 and SK-BR-3 cells expressed Fibulin-2. Expression of Fibulin-2 mRNA and protein in SK-BR-3 Fibulin-2 siRNA group was significantly lower than that in SK-BR-3 NC siRNA group $48 \mathrm{~h}$ after transfection $(\mathrm{P}<0.01)$, while the expression of Fibulin-2 mRNA and protein in MDA-MB-231 Fibulin-2 lentiviral transfection (LAP) group was significantly higher than that in MDA-MB-231 NC LAP group. Compared with the MDA-MB-231 NC LAP group, the cell proliferation, migration and invasion ability of MDA-MB-231 Fibulin-2 LAP group were weakened. The tumor volume and weight of the MDA-MB-231 Fibulin-2 LAP group were significantly lower than those of the MDA-MB-231 NC LAP group. Low
\end{abstract}

Correspondence to: Dr Chaojun Zhang, Department of General Surgery, Navy General Hospital, 6 Fucheng Road, Haidian, Beijing 100048, P.R. China

E-mail: caishun2555@163.com

${ }^{*}$ Contributed equally

Key words: Fibulin-2, breast cancer cells, proliferation, migration, invasion expression of Fibulin-2 is able to promote proliferation, migration and invasion of breast cancer cells, and can reduce the rate of tumor growth in nude mice. Therefore, Fibulin- 2 may be a potential therapeutic target and an indicator of prognosis for breast cancer.

\section{Introduction}

Breast cancer is a common malignancy in women, which has a high incidence rate. Currently, it has become the principal disease affecting women's life and health (1). Moreover, breast cancer is characterized by high migration, which is mainly caused by lymphatic vessels and reflux veins in breasts of women. Usually, tumor spread and migration are detected in most patients at a late stage, leading to poor surgical treatment efficacy, so the death rate of patients is relatively high (2). Based on statistics, it is found that women suffering from breast cancer are getting younger in China, and the incidence and fatality rates of breast cancer are increasing year by year (3). With the development of molecular biology and evidence-based medicine in recent years, the clinical treatment methods for breast cancer mainly include surgical resection, radiotherapy, chemotherapy and targeted therapy (4).

Fibulin-2 is one of the largest isomer proteins in the Fibulin family and also an important component of the extracellular matrix basement membrane and elastic basal fibers. Extracellular matrix is the environment in which cells live. Therefore, Fibulin-2 is capable of affecting cell metabolism, migration, proliferation and differentiation by regulating signaling pathways $(5,6)$. Fibulin-2 is an important component of cells to keep normal physiological activities, which exerts various functions such as regulation of cell metabolism and survival by binding to various extracellular ligands and calcium ions (7). Studies have manifested that knockout of Fibulin-2 gene in mice results in abnormal development and multiple pathological symptoms of mice. Therefore, Fibulin-2 is closely correlated with the normal survival of mice. It was also found that Fibulin-2 plays a key role in the occurrence and development of tumors and is expected to be a new target for overcoming tumors (8-10).

In this study, breast cancer MDA-MB-231, BT483, MCF-7 and SK-BR-3 cell lines was selected and subjected 
to in vitro culture, followed by upregulation and downregulation of Fibulin-2 expression using ribonucleic acid interference (RNAi) technology and lentiviral transfection technique, respectively, expression levels of Fibulin-2 messenger RNA (mRNA) and protein were measured via quantitative real-time reverse transcription polymerase chain reaction (qRT-PCR) and western blotting, respectively, the proliferation ability of breast cancer cells after transfection was detected by Cell Counting Kit-8 (CCK8) assay, and wound healing assay and Transwell chamber assay were performed to detect the impact of Fibulin-2 on the migration and invasion of breast cancer cells, respectively, to study the effects of Fibulin-2 expression on the proliferation, migration and invasion of breast cancer cells, thus providing a basis for research on Fibulin-2 as a therapeutic target for breast cancer.

\section{Materials and methods}

Materials and reagents. Breast cancer MDA-MB-231, BT483, MCF-7 and SK-BR-3 cell lines (the Cell Bank of the Shanghai Institutes for Biological Sciences of the Chinese Academy of Sciences, Shanghai, China), CCK8 kit and Giemsa stain (Sigma-Aldrich; Merck KGaA), Fibulin-2 small interfering RNA (siRNA) (sc-43119, Santa Cruz Biotechnology, Inc.), negative control (NC) siRNA (sc-37007, Santa Cruz Biotechnology, Inc.), Fibulin-2 lentiviral activation particles (LAP) and pLenti-C-Myc-DDK-P2A-Puro Tagged Cloning Vector (cat. nos. RC218622L3V, PS100092, OriGene Technologies, Inc.), Fibulin-2 primary antibody and horseradish peroxidase (HRP)-labeled secondary antibody (Proteintech Group, Inc.), TB Green ${ }^{\mathrm{TM}}$ Fast qPCR Mix (Takara); Roswell Park Memorial Institute (RPMI)-1640 medium and fetal bovine serum (FBS) (Gibco; Thermo Fisher Scientific, Inc.), and TRIzol kit (Invitrogen; Thermo Fisher Scientific, Inc.). Sixteen BALB/C-nu female nude mice, 4-5 weeks old and 15-18 g, were purchased by SPF (Beijing) Biotechnology Co., Ltd. The study was approved by the Ethics Committee of Navy General Hospital (Beijing, China)

Cell culture. MDA-MB-231, BT483, MCF-7 and SK-BR-3 cells were cultured with RPMI-1640 medium containing $10 \% \mathrm{FBS}$ in an incubator at $37^{\circ} \mathrm{C}$ and $5 \% \mathrm{CO}_{2}$. The medium was changed every $24 \mathrm{~h}$. Cells were subjected to digestion and passaged when they were confluent.

Transfection with Fibulin-2 siRNA and Fibulin-2 overexpression lentivirus. SK-BR-3 cells (Fibulin-2 overexpression) were selected as siRNA interference model and MDA-MB-231 cells (expression deletion) as Fibulin-2 overexpression model. Breast cancer cells in the logarithmic growth phase were collected and subjected to digestion process. Then, cells were divided into 4 groups: SK-BR-3 NC siRNA group, SK-BR-3 Fibulin-2 siRNA group, MDA-MB-231 NC LAP group and MDA-MB-231 Fibulin-2 LAP group. Interfering and overexpressing were conducted in accordance with the instructions of Santa Cruz Biotechnology, Inc. siRNA Transfection and LAP Transduction, respectively, establishing Fibulin-2 siRNA-transiently-transfected cell line and Fibulin-2-overexpressed stable cell line for subsequent experiments.
Detection of the effects of siRNA interference and lentivirus overexpression on Fibulin-2 mRNA expression via qRT-PCR. TRIzol assay was applied to extract RNA from cells in each group. Then, the concentration and purity were measured. After that, qualified RNA was selected for reverse transcription that was performed based on the instructions of the reverse transcription kit (Eppendorf). The system was $10 \mu 1$. The specific reaction conditions: $37^{\circ} \mathrm{C}$ for $15 \mathrm{~min}$ and $95^{\circ} \mathrm{C}$ for 5 min, followed by cooling on ice. Thereafter, routine qRT-PCR was conducted, with primer sequences are shown in Table I. $\beta$-actin was selected as the internal reference. The reaction conditions: $94^{\circ} \mathrm{C}$ for $5 \mathrm{~min}$, and then $94^{\circ} \mathrm{C}$ for $30 \mathrm{sec}, 52^{\circ} \mathrm{C}$ for $30 \mathrm{sec}$ and $72^{\circ} \mathrm{C}$ for $30 \mathrm{sec}$, with 40 cycles of amplification in total, followed by $72^{\circ} \mathrm{C}$ for $5 \mathrm{~min}$. Cycle threshold $(\mathrm{Ct})$ value was output from the instrument (Bio-Rad Laboratories, Inc.), and the relative expression level of Fibulin-2 mRNA was calculated using the $2^{-\Delta \mathrm{CT}}$ method.

Determination of the influences of siRNA interference and lentivirus overexpression on Fibulin-2 protein expression through western blotting. Subsequently cells in each group were collected, the total protein was extracted. Then, bicinchoninic acid (BCA) assay was adopted to measure protein concentration, and corresponding loading buffer was added, followed by heating for protein denaturation. Afterwards, $40 \mu \mathrm{g}$ protein was taken from each group for sodium dodecyl sulfate polyacrylamide gel electrophoresis (SDS-PAGE), followed by wet constant current (300 mA) membrane transfer and $1 \mathrm{~h}$ of blocking with $5 \%$ skim milk powder at room temperature. Thereafter, the membrane was incubated with primary antibody (diluted at $1: 1000$ ) at $4^{\circ} \mathrm{C}$ overnight and washed with Tris-buffered saline with Tween-20 ${ }^{\circledR}$ (TBST) 3 times ( $8 \mathrm{~min} /$ time). Then, incubation with secondary antibody (diluted at 1:2,000) at room temperature for $1 \mathrm{~h}$ and washing with TBST 3 times ( $8 \mathrm{~min} /$ time) were performed, followed by dark room development with enhanced chemiluminescence (ECL) and scanning and photographing using a gel imager. With glyceraldehyde-3-phosphate dehydrogenase (GAPDH) as the internal reference, gray analysis and comparison were conducted.

Detection of the effects of siRNA interference and lentivirus overexpression on cell proliferation ability using CCK-8 assay. Cells in each group were inoculated into a 96-well plate at a concentration of $5 \times 10^{4} / \mathrm{ml}$, with $100 \mu \mathrm{l}$ in each well. After cells were routinely cultured for $48 \mathrm{~h}, 20 \mu \mathrm{l}$ CCK 8 was added into each well, followed by $2 \mathrm{~h}$ of culture in an incubator. Then, cells were taken out to measure the absorbance (A) value at $450 \mathrm{~nm}$ using a microplate reader. The white well was used for zero adjustment. Cell proliferation rate was calculated according to the following formula: proliferation rate $(\%)=$ optical density $(\mathrm{OD})$ value in experimental group / OD value in normal control group x $100 \%$.

Determination of the impact of siRNA interference and lentivirus overexpression on cell metastatic ability via wound healing assay. Wound healing assay was carried out to detect the metastatic ability of cells. Before the inoculation of cells, a marking pen was used to mark a transverse line on the back of a 6 -well plate. Then, cells 
Table I. RT-PCR primer sequences.

\begin{tabular}{llr}
\hline Gene & Primer name & Primer sequence \\
\hline$\beta$-actin & Forward primer & 5'-GCTTGGAATGAGACTGCTGA-3' \\
& Reverse primer & 5'-CTGGCCATATCCACCAGAGT-3' \\
Fibulin-2 & Forward primer & 5'-GCAGCTCTTCTCCTGCAAGT-3' \\
& Reverse primer & 5'-CAGACCCCAACTCTGTCCAT-3'
\end{tabular}

were inoculated into the 6-well plate at the appropriate density after they were digested. The next day, cell scratches were made perpendicular to the plate using a $1 \mathrm{ml}$ tip. Then, cell medium was aspirated, and the plate was washed with phosphate-buffered saline (PBS) 3 times. Then, serum-free medium was added, followed by photography, and the plate was placed in an incubator and taken out for photography every $6 \mathrm{~h}$. The experimental results were analyzed based on the collected image data.

Detection of the influence of siRNA interference and lentivirus overexpression on cell invasion capacity through Transwell assay. The invasive ability of cells was detected using Transwell assay. First, Transwell chambers were coated with Matrigel. Then, cells in each group were inoculated into Transwell chambers at a concentration of $4 \times 10^{4} / \mathrm{ml}$, with $100 \mu \mathrm{l}$ per well. Thereafter, $100 \mu \mathrm{l}$ cell suspension and $100 \mu \mathrm{l}$ serum-free medium were evenly added into upper chambers, and lower chambers were added with $500 \mu 1$ medium containing $30 \%$ FBS. After cells were incubated in the incubator for $48 \mathrm{~h}$, chambers were taken out, and then cells were fixed with formaldehyde, stained with crystal violet, photographed by a microscope and analyzed.

Establishment of tumor transplantation model in nude mice. MDA-MB-231 NC LAP and MDA-MB-231 Fibulin-2 LAP cells were cultured in vitro. When the cells were in logarithmic growth phase, cells was subjected to digestion process and counted. The cell suspension was prepared with PBS and the concentration was $1 \times 10^{7} / \mathrm{ml}$. Each nude mouse was inoculated with $0.2 \mathrm{ml}$ cell suspension under the right anterior armpit. The tumor volume was measured every 3 days after inoculation. On the 21 st day, the nude mice were sacrificed and the tumor was taken out and weighed. The mice were kept in cages with controlled temperature, light cycles and humidity $\left(24^{\circ} \mathrm{C}\right.$ and $12 / 12$-h light cycles, $60 \pm 10 \%)$ and free access to water. When the tumor diameter of mice was $10 \mathrm{~mm}$, the mice were anesthetized with $400 \mathrm{mg} / \mathrm{kg}$ chloral hydrate, then tumor tissue was taken and the mice were sacrificed by cervical dislocation. No sign of peritonitis was observed.

Statistical processing. Statistical Product and Service Solutions (SPSS) 19.0 software (IBM Corp.) was used for data processing. All data were expressed as mean \pm standard deviation (SD). t-test was employed for comparisons among groups. $\mathrm{P}<0.05$ indicates that the difference was statistically significant.

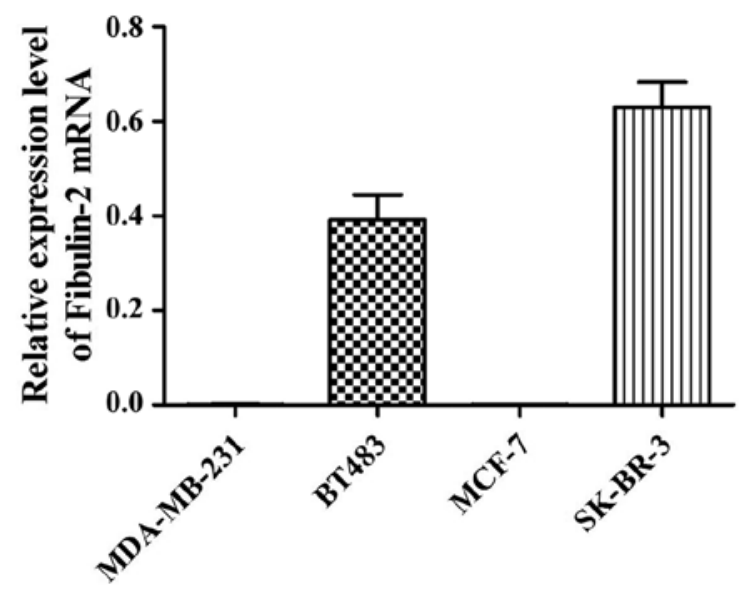

Figure 1. Expression level of Fibulin-2 mRNA in different breast cancer cells detected via qRT-PCR. qRT-PCR, quantitative real-time reverse transcription polymerase chain reaction. mRNA, messenger RNA.

\section{Results}

Fibulin-2 mRNA expression in different breast cancer cells detected via $q R T-P C R$. Fibulin-2 mRNA expression was not detected in MDA-MB-231 and MCF-7 cells, but Fibulin-2 expression was detected in BT483 and SK-BR-3 cells (Fig. 1).

Effects of Fibulin-2 siRNA interference and lentivirus overexpression on the expression of Fibulin-2. The expression of Fibulin-2 was detected by qRT-PCR and western blotting showed that the expression of Fibulin- 2 mRNA and protein in SK-BR-3 Fibulin-2 siRNA group was significantly lower than that in SK-BR-3 NC siRNA group $48 \mathrm{~h}$ after transfection $(\mathrm{P}<0.01)$. The expression of Fibulin-2 mRNA and protein in MDA-MB-231 Fibulin-2 LAP group was significantly higher than that in MDA-MB-231 NC LAP group $(\mathrm{P}<0.01)$ (Fig. 2).

Effect of Fibulin-2 on the proliferation of breast cancer cells. The changes of cell proliferation ability were detected by CCK8 assay showing that the cell proliferation ability of SK-BR-3 Fibulin-2 siRNA group was significantly higher than that of SK-BR-3 NC siRNA group $(\mathrm{P}<0.01)$, while that of MDA-MB-231 Fibulin-2 LAP group was significantly lower than that of MDA-MB-231 NC LAP group $(\mathrm{P}<0.01)$ (Fig. 3).

Influence of Fibulin-2 on the migration of breast cancer cells. The cell migration ability of SK-BR-3 Fibulin-2 siRNA group was significantly higher than that of SK-BR-3 NC siRNA group $(\mathrm{P}<0.01$, while that of MDA-MB-231 Fibulin-2 LAP group 
A

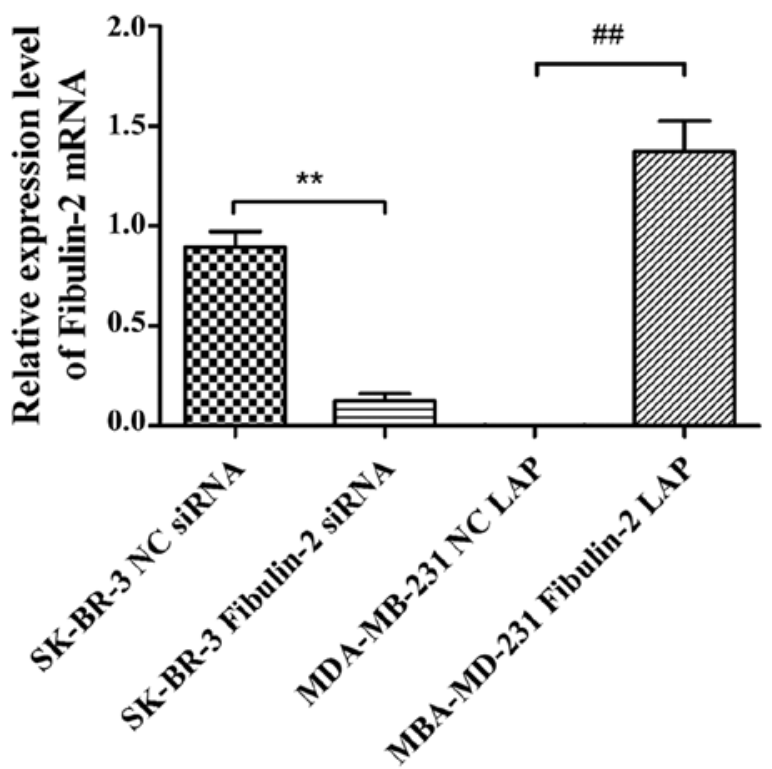

B

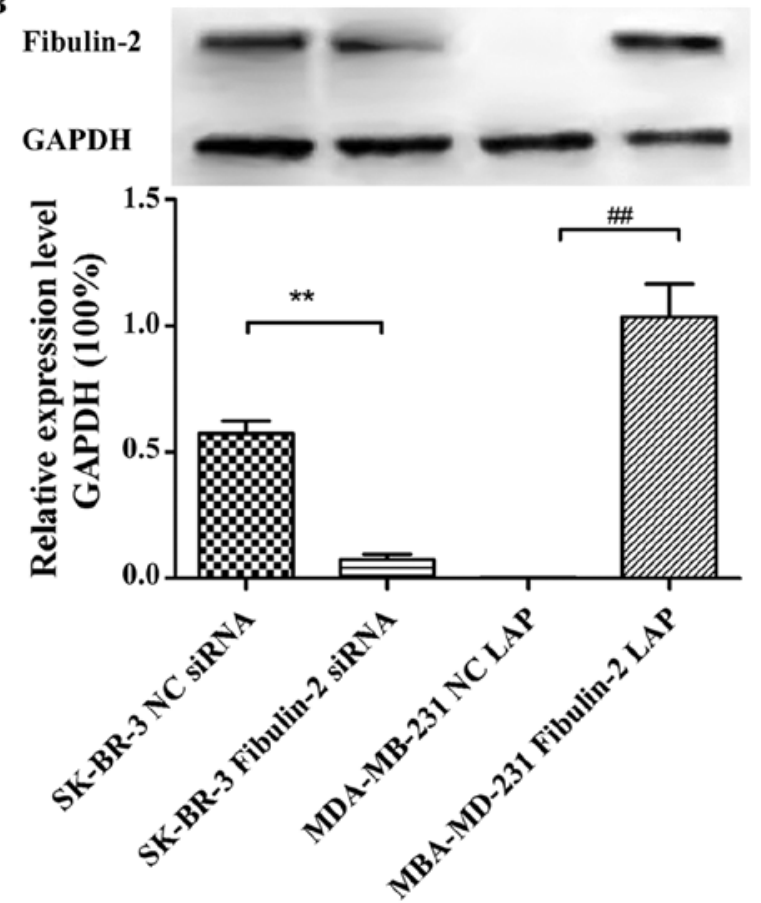

Figure 2. Effects of Fibulin-2 siRNA interference and lentivirus overexpression on the expression of Fibulin-2. (A) Expression of Fibulin-2 in different groups. (B) Expression of GAPDH in different groups. ${ }^{* *} \mathrm{P}<0.01 ;{ }^{\# \#} \mathrm{P}<0.01$. GAPDH, glyceraldehyde-3-phosphate dehydrogenase.

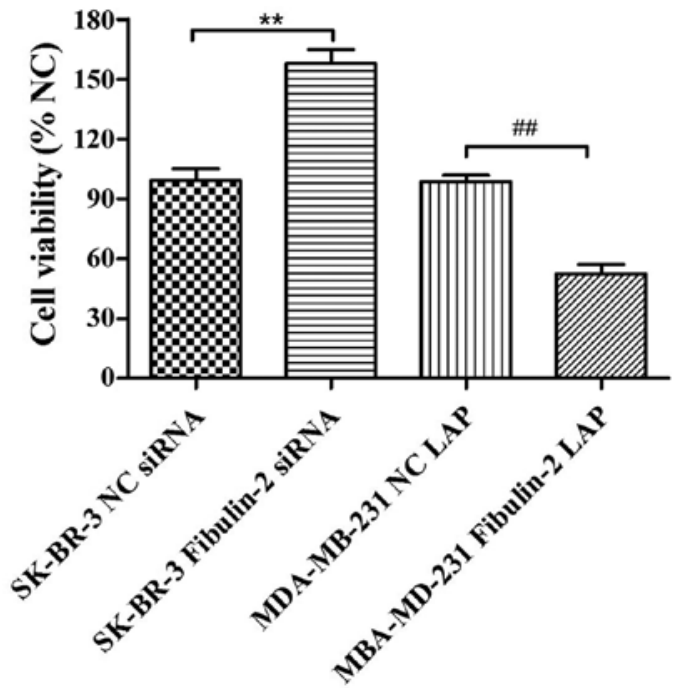

Figure 3. Effect of Fibulin-2 on the proliferation of breast cancer cells. ${ }^{* *} \mathrm{P}<0.01 ;{ }^{\# \#} \mathrm{P}<0.01$.

was significantly lower than that of MDA-MB-231 NC LAP group $(\mathrm{P}<0.01)$ (Fig. 4).

Impact of Fibulin-2 on the invasion of breast cancer cells. The invasive ability of cells in SK-BR-3 Fibulin-2 siRNA group was significantly higher than that in SK-BR-3 NC siRNA group $(\mathrm{P}<0.01)$, while that in MDA-MB-231 Fibulin-2 LAP group was significantly lower than that in MDA-MB-231 NC LAP group $(\mathrm{P}<0.01)$ (Fig. 5).

Effect of Fibulin-2 on tumor growth in nude mice. Compared with MDA-MB-231 NC LAP group, the tumor weight of nude mice in MDA-MB-231 Fibulin-2 LAP group was significantly lower than that in MDA-MB-231 NC LAP group $(\mathrm{p}<0.01)$, and the tumor growth and tumor volume in MDA-MB-231 Fibulin-2 LAP group were significantly lower than those in MDA-MB-231 NC LAP group ( $<<0.01)$ (Fig. 6).

\section{Discussion}

Breast cancer has become a growing threat to women's health, and its age of onset is getting younger. Furthermore, its incidence rate ranks first among urban female malignant tumors. With the increase in the incidence rate of breast cancer, the side effects of chemotherapy drugs and the huge demand of clinical treatment, increasing number of researchers pay attention to the exploration of new mechanisms and targets of breast cancer (11).

Remodeling of extracellular matrix can be achieved by the synthesis and degradation of matrix proteins. A study manifested that extracellular matrix is closely associated with the differentiation, migration and migration of most tumors. After remodeling, there are obvious changes in physical properties and spatial structure of extracellular matrix proteins. Therefore, the characteristics and molecules relating to extracellular matrix remodeling can be used as important indicators for early diagnosis and clinical staging of tumors (12). Another study revealed that Fibulin-2 protein, as an important component of the extracellular matrix, is closely correlated with the occurrence of cancer and plays a dual role in the occurrence and development of tumors, i.e., it inhibits tumorigenesis under certain conditions and also promotes the growth of tumor cells under such conditions (13). Studies have revealed that Fibulin-2 suppresses the migration and invasion of tumors such as nasopharyngeal carcinoma and astrocytoma, 

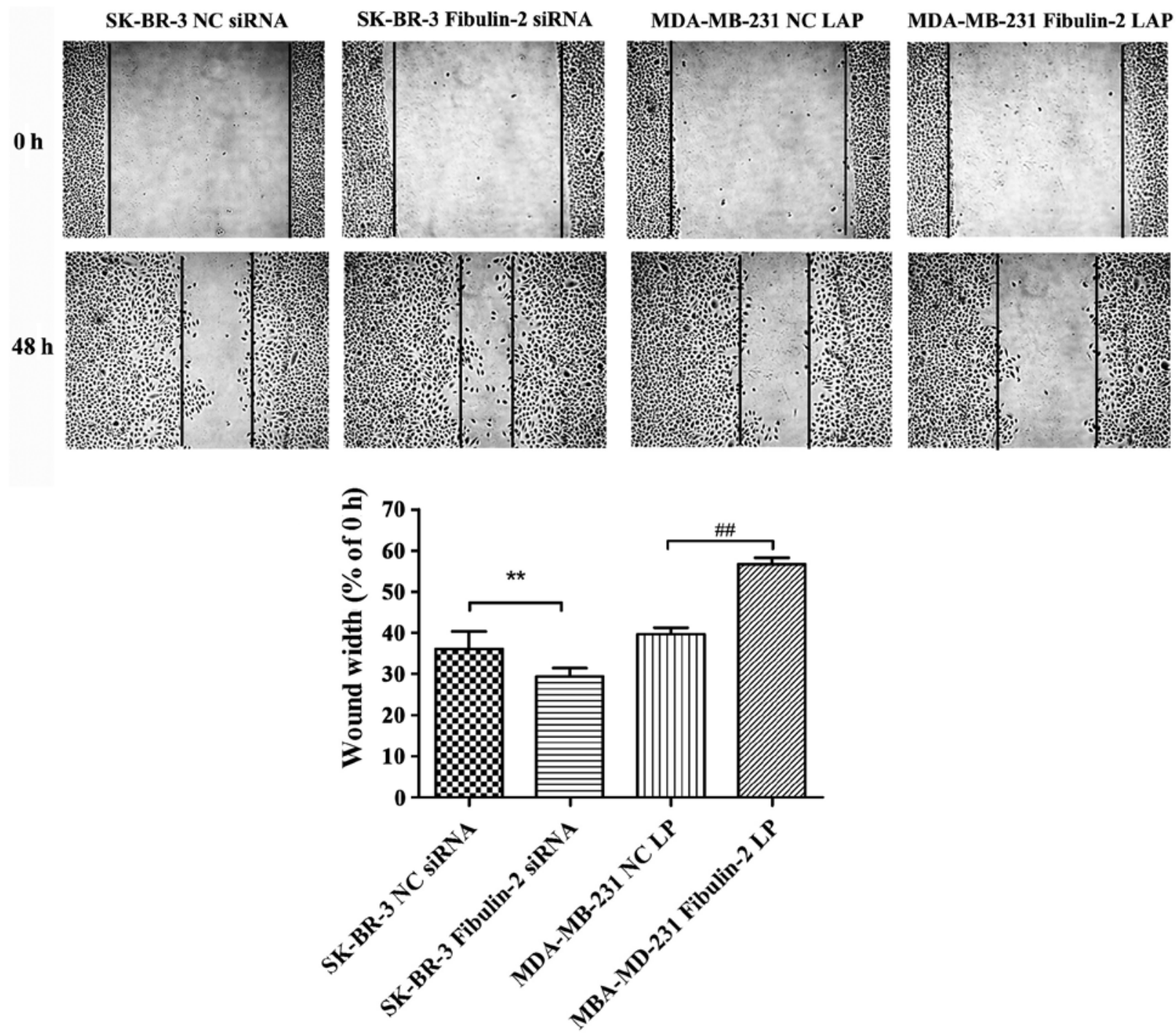

Figure 4. Influence of Fibulin-2 on the migration of breast cancer cells detected through CCK8 assay. CCK8, Cell Counting Kit-8. Comparison between SK-BR-3 Fibulin-2 siRNA group and SK-BR-3 NC siRNA group, ${ }^{* *} \mathrm{P}<0.01$. Comparison between MDA-MB-231 Fibulin-2 LAP group and MDA-MB-231 NC LAP group, ${ }^{\# /} \mathrm{P}<0.01$.

\section{SK-BR-3 NC siRNA}

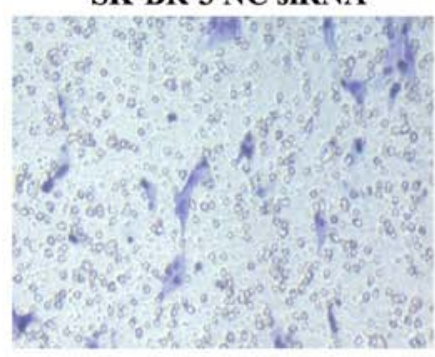

MDA-MB-231 NC LAP

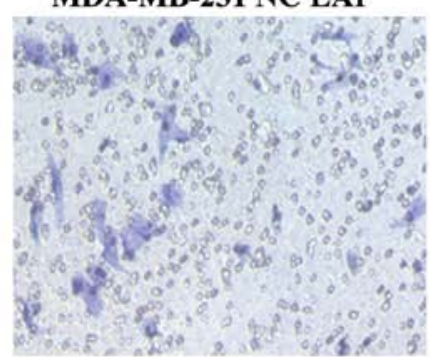

\section{SK-BR-3 Fibulin-2 siRNA}

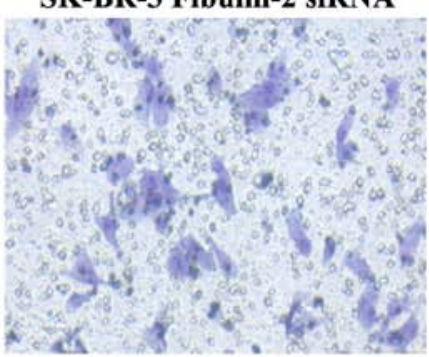

MDA-MB-231 Fibulin-2 LAP

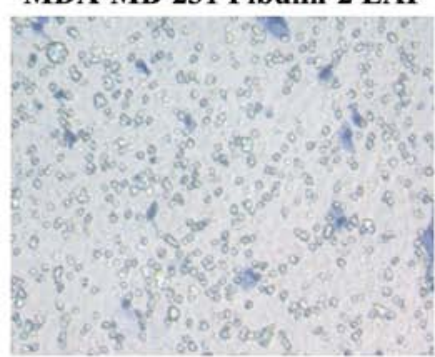

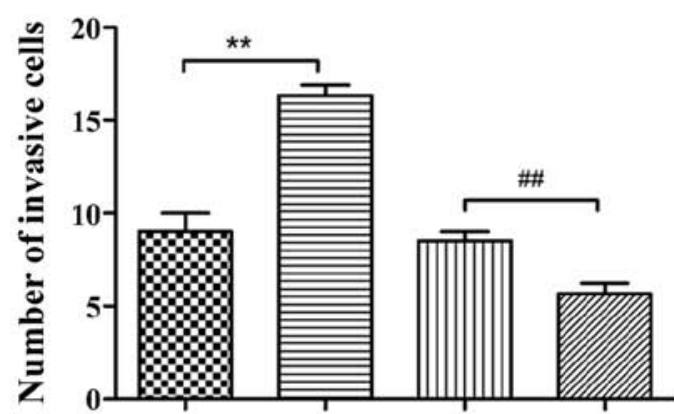

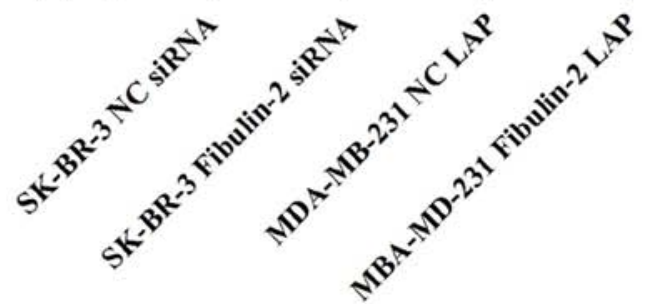

Figure 5. Impact of Fibulin-2 on the invasion of breast cancer cells detected via Transwell assay. ${ }^{* *} \mathrm{P}<0.01 ;{ }^{\# \#} \mathrm{P}<0.01$. 
A

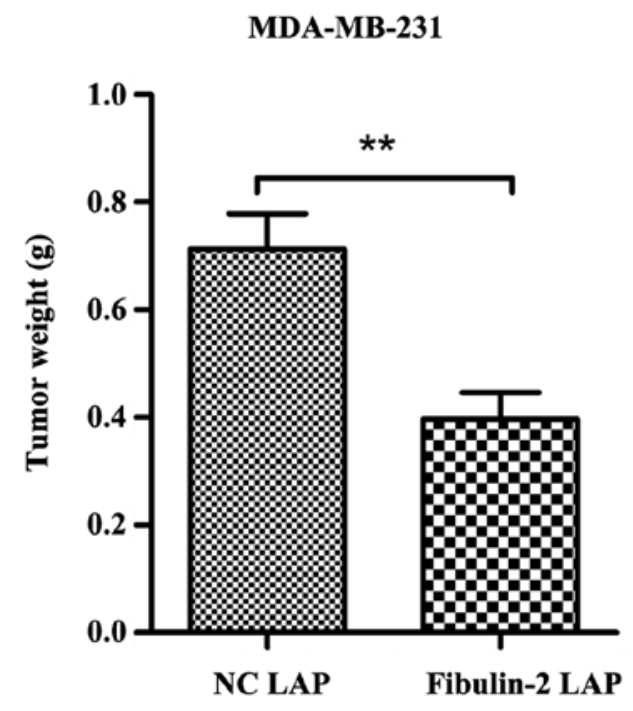

B

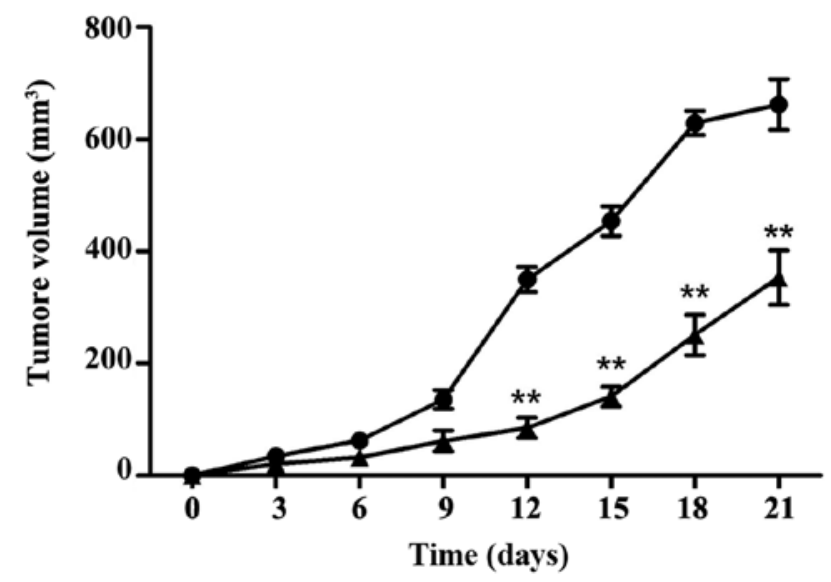

Figure 6. Effect of Fibulin-2 on tumor weight and volume in nude mice. ${ }^{* *} \mathrm{P}<0.01$. (A) Tumor weight in different groups. (B) Tumor volume in different groups.

but it promotes migration and invasion of tumor cells in lung adenocarcinoma (14-16).

A recent study found that there is no expression of Fibulin-2 in breast cancer patients, whereas normal in paracancerous tissues of breast cancer, which confirmed that Fibulin-2 can stabilize the basement membrane, and its downregulation is related to the loss of basement membrane and early invasion (17). Tan et al (18) showed that Fibulin-2 was expressed in breast ducts and perivascular tissues in normal breast tissues. In malignant breast tissues, collagen IV was integrated around ducts, while Fibulin-2 was not fully expressed around collagen IV, which showed that Fibulin-2 was involved in tumor invasion and migration. Moreover, it has been shown that Fibulin2 is a new matrix of ADAMTS5 and the degradation of Fibulin2 is associated with the enhancement of the invasion potential of SKBR3 cells, and this proteolytic hydrolysis can alter the cell microenvironment and affects the balance between tumor and anti-tumor effects associated with Fibulin2 and ADAMTS metalloproteinase (19).

Studies have verified that Fibulin-2 is capable of promoting the development and progression of lung cancer and pancreatic cancer, with different expression levels in lung cancer, pancreatic cancer and adjacent normal tissues and a mouse lung cancer cell line. It was discovered in a previous study that Fibulin-2 is highly expressed in human lung adenocarcinoma extracellular matrix and metastatic lung adenocarcinoma mouse tumor cell lines. A study found that knockout of Fibulin-2 leads to small tumor growth and low migration rate by constructing stable mouse lung adenocarcinoma tumor cells without Fibulin-2 gene and establishing a nude-mouse model of transplantation. Research at the cellular level discovered that the migration and invasion ability of tumor cells is weakened after knockdown of Fibulin-2 gene, and Fibulin-2 promotes cell collagen cross-linking and adhesion in lung tumor (20-22). Studies have confirmed that Fibulin-2 is overexpressed in different solid tumor tissues, fully proving that Fibulin-2 plays a vital role in the occurrence and development of tumors (23).
In this investigation, a variety of breast cancer cells were cultured in vitro. qRT-PCR results showed that MDA-MB-231 and MCF-7 cells did not express Fibulin-2, while BT483 and SK-BR-3 cells expressed Fibulin-2. Expression of Fibulin-2 was upregulated and downregulated using RNAi technology and lentiviral transfection techniques, respectively, and the expression levels of Fibulin-2 mRNA and protein were evaluated via qRT-PCR and western blotting, respectively. The results showed that the expression of Fibulin-2 mRNA and protein in SK-BR-3 Fibulin-2 siRNA group was significantly lower than that in SK-BR-3 NC siRNA group, while the expression of Fibulin-2 mRNA and protein in MDA-MB-231 Fibulin-2 LAP group was significantly higher than that in MDA-MB-231 NC LAP group $48 \mathrm{~h}$ after transfection. The results of cell proliferation, Scratch and Transwell tests showed that compared with SK-BR-3 NC siRNA group, the ability of cell proliferation, migration and invasion in SK-BR-3 Fibulin-2 siRNA group was enhanced. Compared with MDA-MB-231 NC LAP group, the ability of cell proliferation, migration and invasion in MDA-MB-231 Fibulin-2 LAP group was reduced. It was found that the tumor weight and volume of MDA-MB-231 Fibulin-2 LAP group decreased significantly after upregulating the expression of Fibulin-2 in nude mice.

In conclusion, this study demonstrates that Fibulin-2 expression can greatly affect the proliferation, migration and invasion of human breast cancer cell lines. Therefore, Fibulin-2 may be a potential therapeutic target and a prognostic index for breast cancer.

\section{Acknowledgements}

Not applicable.

\section{Funding}

No funding was received. 


\section{Availability of data and materials}

The datasets used and/or analyzed during the current study are available from the corresponding author on reasonable request.

\section{Authors' contributions}

$\mathrm{XZ}$ wrote the manuscript. XZ and LD performed PCR and western blot. YZ and HZ were responsible for cell culture and transfection. XY and CZ contributed to wound healing assay and Transwell assay. All authors read and approved the final manuscript.

\section{Ethics approval and consent to participate}

The study was approved by the Ethics Committee of Navy General Hospital (Beijing, China).

\section{Patient consent for publication}

Not applicable.

\section{Competing interests}

The authors declare that they have no competing interests.

\section{References}

1. Ducros E, Berthaut A, Mirshahi P, Lemarchand S, Soria J, Legeais JM and Mirshahi M: Expression of extracellular matrix proteins fibulin-1 and fibulin-2 by human corneal fibroblasts. Curr Eye Res 32: 481-490, 2007.

2. Wight TN: The extracellular matrix and atherosclerosis. Curr Opin Lipidol 6: 326-334, 1995.

3. Serra N, Rosales R, Masana L and Vallvé JC: Simvastatin increases fibulin-2 expression in human coronary artery smooth muscle cells via RhoA/Rho-kinase signaling pathway inhibition. PLoS One 10: e0133875, 2015.

4. Aspberg A, Adam S, Kostka G, Timpl R and Heinegård D: Fibulin-1 is a ligand for the C-type lectin domains of aggrecan and versican. J Biol Chem 274: 20444-20449, 1999.

5. Olin AI, Mörgelin M, Sasaki T, Timpl R, Heinegård D and Aspberg A: The proteoglycans aggrecan and Versican form networks with fibulin-2 through their lectin domain binding. J Biol Chem 276: 1253-1261,2001.

6. Kwan ML, Kushi LH, Weltzien E, Maring B, Kutner SE, Fulton RS, Lee MM, Ambrosone CB and Caan BJ: Epidemiology of breast cancer subtypes in two prospective cohort studies of breast cancer survivors. Breast Cancer Res 11: R31, 2009.

7. Siegel R, Naishadham D and Jemal A: Cancer statistics, 2013. CA Cancer J Clin 63: 11-30, 2013.

8. Chen WQ, Zeng HM, Zheng RS, Zhang SW and He J: Cancer incidence and mortality in China, 2007. Chin J Cancer Res 24: $1-8,2012$.
9. Werb Z: ECM and cell surface proteolysis: Regulating cellular ecology. Cell 91: 439-442, 1997.

10. Wu TS and Hammond GL: Naturally occurring mutants inform SHBG structure and function. Mol Endocrinol 28: 1026-1038, 2014.

11. Shamay M, Barak O and Shaul Y: HBXAP, a novel PHD-finger protein, possesses transcription repression activity. Genomics 79: 523-529, 2002

12. Whiteaker JR, Zhang H, Zhao L, Wang P, Kelly-Spratt KS, Ivey RG, Piening BD, Feng LC, Kasarda E, Gurley KE, et al: Integrated pipeline for mass spectrometry-based discovery and confirmation of biomarkers demonstrated in a mouse model of breast cancer. J Proteome Res 6: 3962-3975, 2007.

13. Hill VK, Hesson LB, Dansranjavin T, Dallol A, Bieche I, Vacher S, Tommasi S, Dobbins T, Gentle D, Euhus D, et al: Identification of 5 novel genes methylated in breast and other epithelial cancers. Mol Cancer 9: 51, 2010.

14. Fontanil T, Rúa S, Llamazares M, Moncada-Pazos A, Quirós PM, García-Suárez O, Vega JA, Sasaki T, Mohamedi Y, Esteban MM, et al: Interaction between the ADAMTS-12 metalloprotease and fibulin-2 induces tumor-suppressive effects in breast cancer cells Oncotarget 5: 1253-1264, 2014.

15. Tian H, Liu J, Chen J, Gatza ML and Blobe GC: Fibulin-3 is a novel TGF- $\beta$ pathway inhibitor in the breast cancer microenvironment. Oncogene 34: 5635-5647, 2015.

16. Chan SW, Lim CJ, Guo K, Ng CP, Lee I, Hunziker W, Zeng $\mathrm{Q}$ and Hong W: A role for TAZ in migration, invasion, and tumorigenesis of breast cancer cells. Cancer Res 68: 2592-2598, 2008.

17. Ibrahim AM, Sabet S, El-Ghor AA, Kamel N, Anis SE, Morris JS and Stein T: Fibulin-2 is required for basement membrane integrity of mammary epithelium. Sci Rep 8: 14139, 2018.

18. Tan H, Zhang J, Fu D and Zhu Y: Loss of fibulin-2 expression is involved in the inhibition of breast cancer invasion and forms a new barrier in addition to the basement membrane. Oncol Lett 14: 2663-2668, 2017.

19. Fontanil T, Álvarez-Teijeiro S, Villaronga MÁ, Mohamedi Y, Solares L, Moncada-Pazos A, Vega JA, García-Suárez O, Pérez-Basterrechea M, García-Pedrero JM, et al: Cleavage of Fibulin-2 by the aggrecanases ADAMTS-4 and ADAMTS-5 contributes to the tumorigenic potential of breast cancer cells. Oncotarget 8: 13716-13729, 2017.

20. Dunwell TL, Hesson LB, Pavlova T, Zabarovska V, Kashuba V, Catchpoole D, Chiaramonte R, Brini AT, Griffiths M, Maher ER, et al: Epigenetic analysis of childhood acute lymphoblastic leukemia. Epigenetics 4: 185-193, 2009.

21. Mesri EA, Cesarman E and Boshoff C: Kaposi's sarcoma and its associated herpesvirus. Nat Rev Cancer 10: 707-719, 2010.

22. Alcendor DJ, Knobel S, Desai P, Zhu WQ and Hayward GS: KSHV regulation of fibulin-2 in Kaposi's sarcoma: Implications for tumorigenesis. Am J Pathol 179: 1443-1454, 2011.

23. Senapati S, Gnanapragassam VS, Moniaux N, Momi N and Batra SK: Role of MUC4-NIDO domain in the MUC4-mediated metastasis of pancreatic cancer cells. Oncogene 31: 3346-3356, 2012.

This work is licensed under a Creative Commons Attribution-NonCommercial-NoDerivatives 4.0 International (CC BY-NC-ND 4.0) License. 